\title{
Chronobiology of sepsis. I. Relationship between sepsis course and urinary excretion of 6-sulfatoxymelatonin
}

Acuña Fernández C., Solera Marín J., Acuña Castroviejo D., Darias Delbey B., Nodarse Medina J., Rusanova.

Hospital Universitario de Canarias- Tenerife, Dept of Anaesthesiology, San Cristobal de la Laguna, Spain, Universidad de Granada, Centro de Investigación Biomédica, Granada, Spain

Background and Goal of Study: Sepsis courses with a shift of the circadian system that affects the melatonin production, which correlated with sleep/wake dysfunction. Melatonin administration recovered mice from septic shock and multiorgan failure, increasing their survival. In septic patients, melatonin administration significantly improved the circadian clock alteration. Moreover, melatonin reduction may be related to the oxidative stress and immune response during sepsis. The aim of this study was to analyze urinary excretion of 6-sulfatoxymelatonin (6-SM), the main metabolite of melatonin, and to compare it with the IL-1 $\beta$, IL-6, IL-8, TNF $\alpha$, IL-10 levels, and oxidative status during sepsis.

Materials and Methods: Healthy subjects, non-septic controls and septic patients were evaluated. 6-SM was measured by a commercial ELISA kit; plasma cytokines were determined with Affimetrix's ProcartaPlex Simplex Kits, and plasma lipid peroxidation (LPO) and protein oxidation (AOPP) were spectrophotometrically measured. Hematological and biochemical data, and clinical scores of the patients, were analyzed.

Results and Discussion: Proinflammatory cytokines, LPO, and AOPP increased during sepsis and normalized in patients recovered from sepsis. The urinary 6-SM decreased in sepsis and increased when the patients recovered from sepsis, suggesting changes in the synthesis and/or metabolization of melatonin and, thus, changes in the circadian profile of melatonin. 6-SM excretion correlates negatively with SOFA and positively
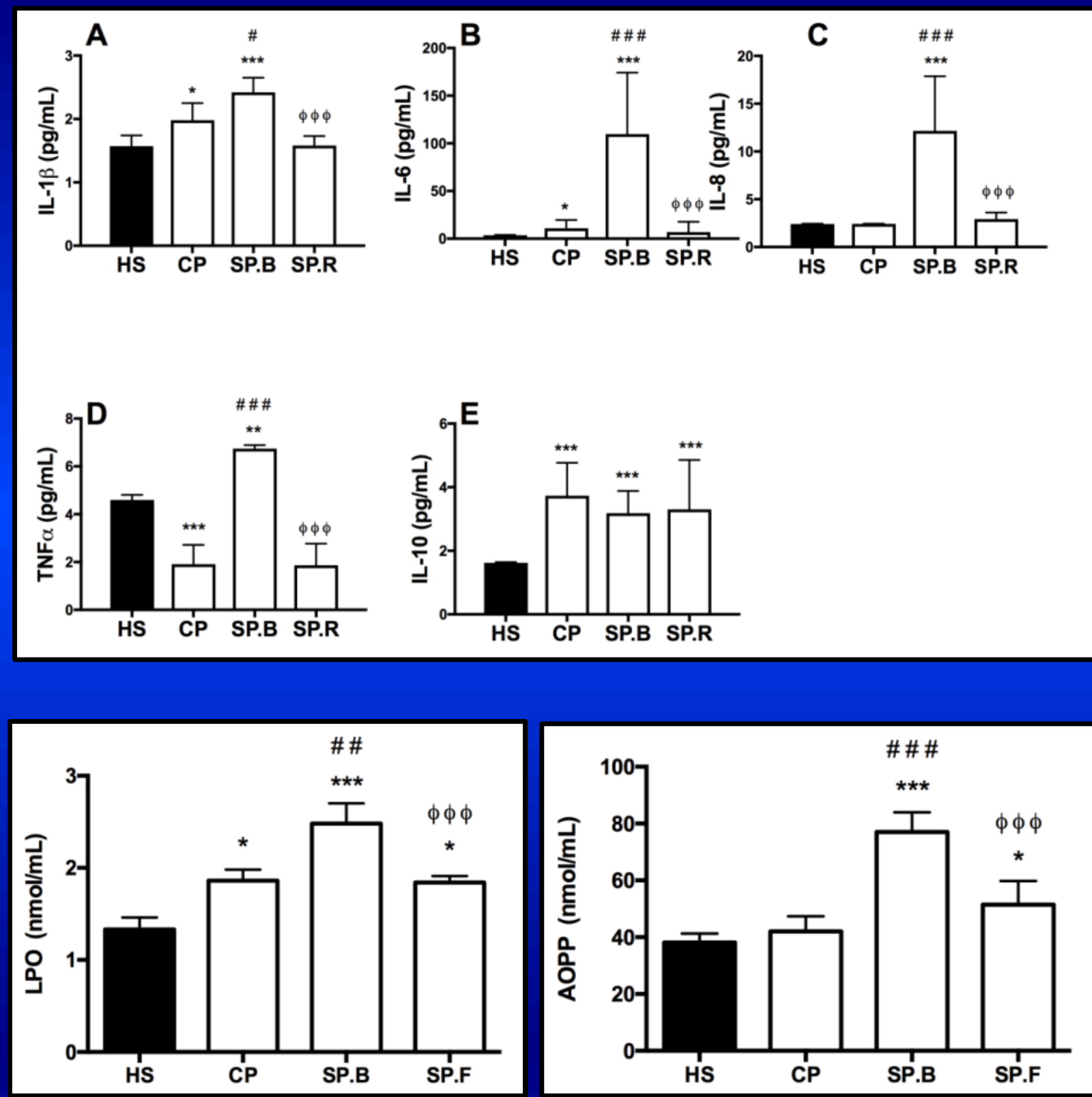

This research has been partially supported by grants RD12/0043/0005 and PI13-00981 from 494 the ISCIII, Spain, and from Pfizer S.L.U, Madrid, Spain.
The significant increased levels of melatonin that accompanies the recovery of sepsis, together with the normalization of the pro-inflammatory cytokines levels and of the hyperoxidative status of septic patients once they were recovered from sepsis, may also support a beneficial effect of melatonin on the sepsis course, as it was demonstrated in both experimental and clinical endotoxemia.
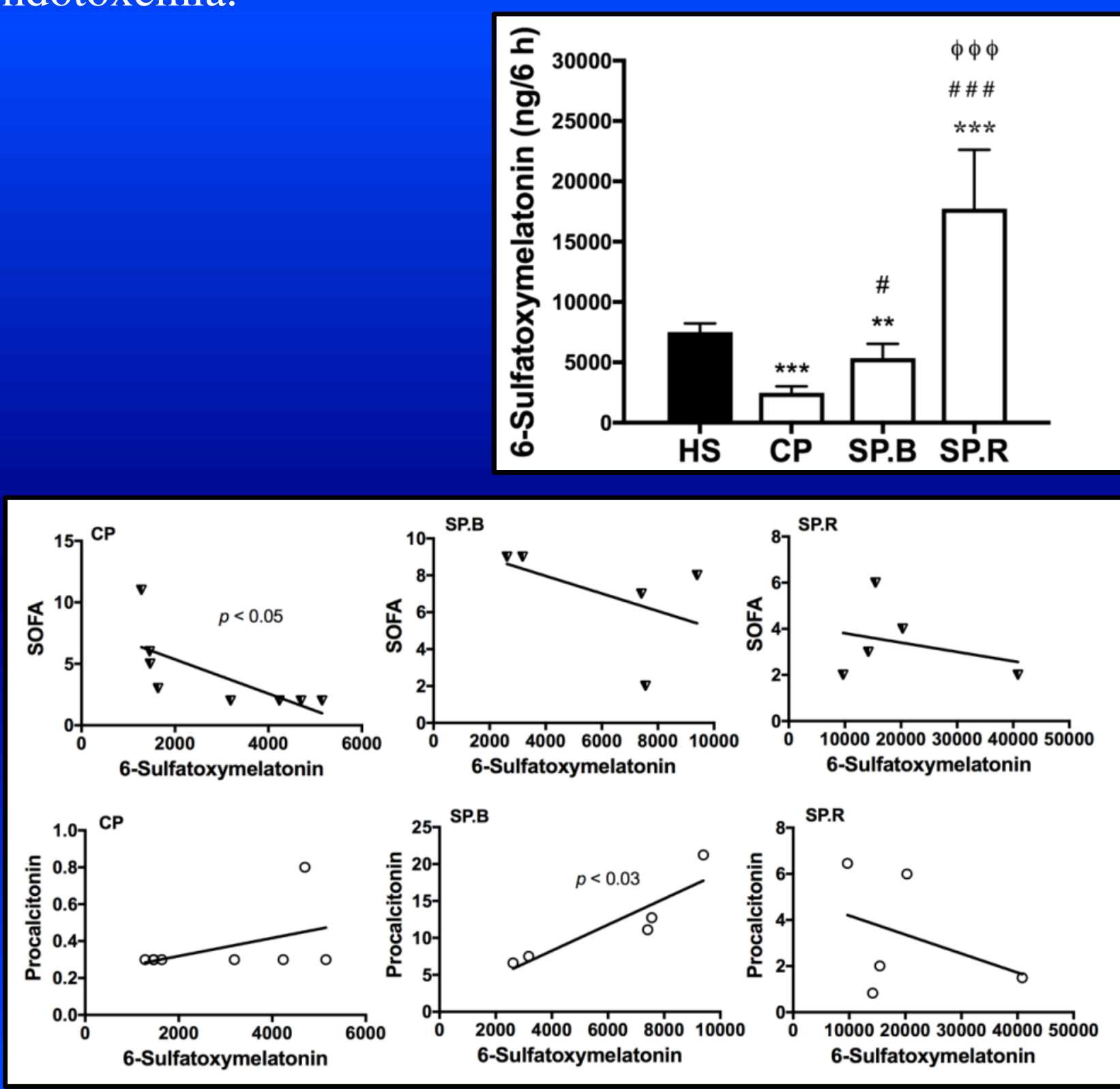

\begin{tabular}{lccc}
\hline Parameter & CP & SP.B & SP.R \\
\hline SAPS & $62.93 \pm 2.96$ & $79.95 \pm 8.31^{*}$ & \\
SOFA & $3.35 \pm 1.05$ & $6.10 \pm 1.16^{* *}$ & $3.46 \pm 0.66^{* *}$ \\
$\mathrm{PaO} 2 / \mathrm{FiO} 2$ & $323.30 \pm 25.09$ & $293.40 \pm 31.89$ & $305.38 \pm 31.46$ \\
$\mathrm{NE}, \mu \mathrm{g} / \mathrm{kg} / \mathrm{min}$ & $<0.3 \pm 0.05$ & $0.53 \pm 0.08^{*}$ & $0.00^{* * *, \mu *}$ \\
$\mathrm{SBP}, \mathrm{mm} \mathrm{Hg}$ & $122.75 \pm 4.44$ & $108.28 \pm 3.78^{*}$ & $136.23 \pm 7.59^{* *}$ \\
$\mathrm{DBP}, \mathrm{mm} \mathrm{Hg}$ & $69.70 \pm 4.03$ & $60.36 \pm 2.42 \#$ & $69.57 \pm 0.92^{*}$ \\
$\mathrm{HR}, \mathrm{bpm}$ & $89.52 \pm 6.74$ & $105.82 \pm 8.81^{*}$ & $92.42 \pm 6.77^{*}$ \\
$\mathrm{RR}, \mathrm{bpm}$ & $13.25 \pm 0.36$ & $14.90 \pm 1.37$ & $13.71 \pm 0.71$ \\
Glasgow & $15.00 \pm 0.001$ & $7.11 \pm 2.40^{m *}$ & $15.00 \pm 0.001^{* * *}$ \\
Fever, ${ }^{\circ} \mathrm{C}$ & $36.06 \pm 0.05$ & $36.66 \pm 0.21$ & $36.00 \pm 0.001$ \\
Urine volume, $\mathrm{mL} / 10 \mathrm{~h}$ & $515.96 \pm 80.53$ & $399.28 \pm 111.92^{*}$ & $613.95 \pm 251.22^{*}$ \\
\hline
\end{tabular}

Conclusions: Our data suggest that the existence of a negative correlation between sepsis and melatonin production, which reflects an alteration in the circadian system, supporting antiseptic properties of the indoleamine elsewhere reported. 